\title{
Optically driven transport into a living cell
}

\section{AUTHOR(S):}

Kubo, K; Ichikawa, M; Yoshikawa, K; Koyama, Y; Niidome, T; Yamaoka, T; Nomura, SIM

\section{CITATION:}

Kubo, K ... [et al]. Optically driven transport into a living cell. APPLIED PHYSICS LETTERS 2003, 83(12): 2468-2470

\section{ISSUE DATE:}

2003-09-22

URL:

http://hdl.handle.net/2433/49850

\section{RIGHT:}

Copyright 2003 American Institute of Physics. This article may be downloaded for personal use only. Any other use requires prior permission of the author and the American Institute of Physics. 


\title{
Optically driven transport into a living cell
}

\author{
Koji Kubo, Masatoshi Ichikawa, and Kenich Yoshikawa ${ }^{a)}$ \\ Department of Physics, Graduate School of Science, Kyoto University \& CREST, Kyoto 606-8502, Japan \\ Yoshiyuki Koyama \\ Department of Home Economics, Otsuma Women's University, Tokyo 102-8357, Japan
}

Takuro Niidome

Department of Materials Science, Graduate School of Science and Technology, Nagasaki University, Nagasaki 852-8521 Japan

Tetsuji Yamaoka

Department of Polymer Science and Engineering, Kyoto Institute of Technology, Kyoto 600-8585, Japan

Shin-Ichirou M. Nomura

Institute of Biomaterials and Bioengineering, Tokyo Medical and Dental University, Tokyo 101-0062, Japan

(Received 5 May 2003; accepted 28 July 2003)

\begin{abstract}
We report a method for transporting foreign substances into a desired living cell through the use of laser trapping. A single giant DNA molecule can be delivered into a cytoplasmic space if the DNA is folded into a compact state. We also find that microparticles of zeolite are very effective as a vehicle for transferring foreign compounds into a living cell by optical manipulation. () 2003 American Institute of Physics. [DOI: 10.1063/1.1613356]
\end{abstract}

The transport of specific foreign substances into a targeted cell is an indispensable procedure in medical science and genetic engineering. However, the only method that is currently available for selective transport into individual cells is micro-injection using a micropipette. ${ }^{1,2}$ For example, in genetic engineering, the insertion of chromatin from a differentiated somatic cell into an oocyte using a micropipette is a widely used technique to obtain cloned cells. ${ }^{3} \mathrm{~A}$ similar technique is used in fertility treatment. Although the micropipette method is a powerful tool, great skill is necessary for successful manipulation. As an alternative method, a high-power laser has been applied in cell biology, where a focused laser is used to make a pore on a cell membrane by local heating. ${ }^{4,5}$ With this method, foreign species in the external solution flow into the cell in a passive manner. In the present study, we applied the methodology of laser trapping to the transport of a foreign substance into a desired cell, as a potentially automated procedure. ${ }^{6}$

The infrared laser used for optical trapping was a Nd:yttrium-aluminum-garnet laser (SL902T, Spectron), with a $\mathrm{TEM}_{00}$ beam at a wavelength of $1064 \mathrm{~nm}$. The laser beam was reflected by a dichroic mirror, and focused by an objective lens (Nikon, $\times 100$, numerical aperature $=1.30$ ) with a microscope (Nikon, TE-300). The beam power was $100-500 \mathrm{~mW}$, as calibrated just before the objective lens of the microscope by a laser power meter (Neoark, PM-345). Experiments on optical delivery and measurements of the trapping efficiency with various particles were performed on the earlier laser trap microscope with a micro-motion horizontal stage (SIGMAKOKI). Ginkgo leaf cells and cabbage protoplasts were used as the recipients. The cabbage protoplasts were prepared by a modified enzyme method using an

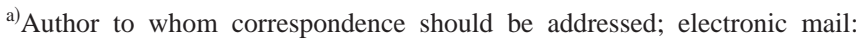
yoshikaw@scphys.kyoto-u.ac.jp
}

enzyme cocktail containing $15 \mathrm{~g} / \mathrm{l}$ Cellulase ONOZUKA (Yakult, Co. Ltd.), 2 g/l Pectoliase Y-23 (Kikkoman, Co. Ltd.), $3 \mathrm{mM}$ MES [2-( $N$-Morpholino) ethanesulfonic acid], $10 \mathrm{mM} \mathrm{CaCl}_{2} 2 \mathrm{H}_{2} \mathrm{O}$ and $0.5 \mathrm{M}$ sorbitol. $^{7}$ The delivered substances were double-stranded T4DNA (Nippon Gene) and the calcium-detecting fluorescent dye fura-2 (Molecular Probes). The multivalent cation PEG-A, a polyethylene glycol derivative with 8.3 amino-pendent groups $(\mathrm{MW}=3900)$ was synthesized as reported previously. ${ }^{8}$ T4 DNA molecules, $0.6 \mu \mathrm{M}$ in base pairs, were collapsed into a compact state by PEG-A (6 $\mu \mathrm{M}$ in amino group concentration). As a delivery vehicle to promote a higher trapping efficiency, we used zeolite particles $\left[\mathrm{Na}_{56}\left(\mathrm{Al}_{56} \mathrm{Si}_{36} \mathrm{O}_{384}\right) \cdot 250 \mathrm{H}_{2} \mathrm{O}\right.$, Toyosoda, Co. Ltd.] ground with a mortar and pestle as a carrier.

Table I compares the trapping efficiencies for different objects: a polystyrene bead with a radius of $0.1 \mathrm{~mm}$ a ground zeolite particle (radius of around $0.2 \mu \mathrm{m}$ ) and a folded compact DNA ( hydrodynamic radius $)^{9}$ under $200 \mu \mathrm{M}$ spermidine. In this table, the trapping efficiency $Q$ is represented as $F=n Q P / c$, where $F, n, P$, and $c$ are the trapping force, refractive index of the surrounding medium, beam power, and light velocity, respectively. The trapping efficiencies were evaluated based on the drag speed of trapped objects under the assumption of Stokes friction. ${ }^{10}$

Figure 1 shows the injection of a single T4 DNA molecule into a ginkgo leaf cell by laser transport. In this case,

TABLE I. Trapping efficiency of submicrometer-sized particles. ${ }^{a}$

\begin{tabular}{lc}
\hline \hline Trapping efficiency $Q\left(10^{-3}\right)^{\mathrm{c}}$ & \\
\hline Compact T4DNA & \\
Fluorescent bead & $0.075 \pm 0.025$ \\
Zeolite particle & $0.80 \pm 0.10$ \\
\hline
\end{tabular}

${ }^{a}$ The medium values of 36 experimental trials are given.

${ }^{\mathrm{b}}$ DNA was compacted by spermidine.

${ }^{\mathrm{c}}$ The extinction ratio of trapping laser was assumed as 0.5 . 

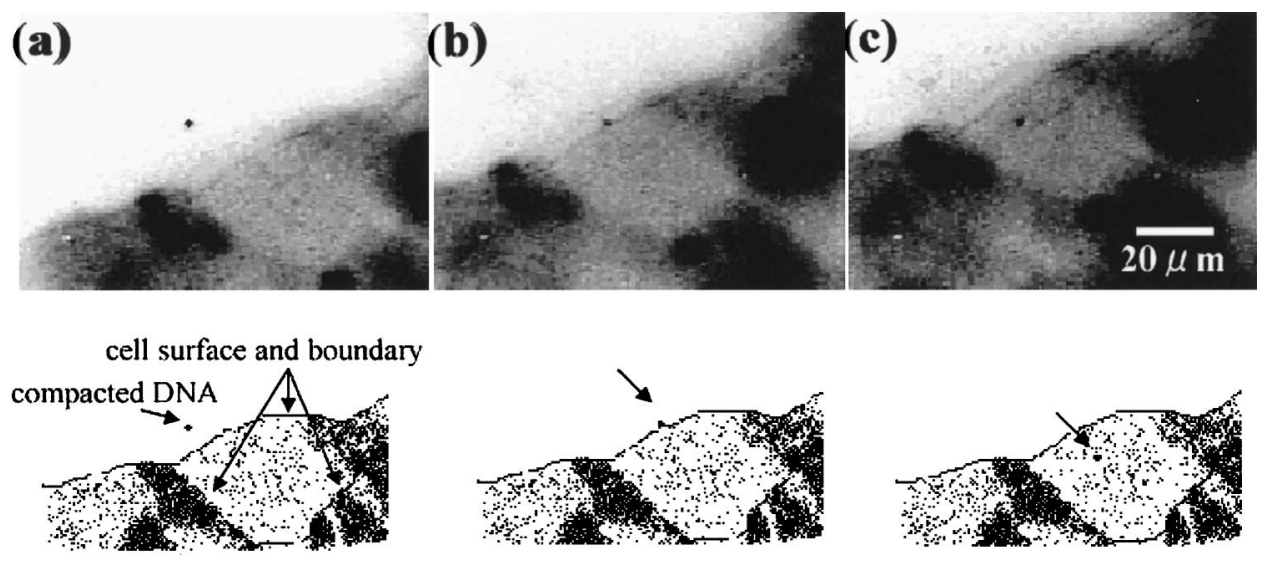

T4 DNA is folded into a compact state by forming a complex with PEG-A. It has been reported that individual giant DNA molecules can be optically trapped when they are folded into a compact state using suitable condensing agents. ${ }^{11}$ The ability to trap DNA molecules stably suggests that it may be possible to apply optical manipulation to the microinjection of DNA into living cells. However, in experiments on compact DNAs, we found that the probability of the successful transport of DNA into a cell was not high (only a few $\%$ in several trials). The difficulty in these experiments is due to the weak optical force for grasping compact DNA, as indicated in Table I.

Therefore, we sought a better method for optically driven transport into a living cell. As shown in Table I, the ability of laser-tweezers to grasp a zeolite particle is much greater than its ability to grasp compact DNA molecules. The higher efficiency for zeolite is based on its permittivity for $1064 \mathrm{~nm}$ light. Although bigger zeolite particles, larger than several microns, have higher trapping efficiencies, they often absorb laser beam photons, which induces flashing evaporation of the medium. Thus, we used micron-sized particles for optical delivery.

Zeolite particles can hold foreign chemicals in their fine pores. Figure 2 shows the transport of a zeolite particle doped with a fluorescent dye, fura-2, into a protoplast cell. After the particle is delivered, emission from fura-2 complexed with intracellular calcium ion is observed [Fig. 2(b)], indicating that the particle is indeed transferred into the cytoplasmic space.

This optically driven transport method may have many practical applications. Compared to the current methodologies, transport by laser trapping should cause less damage to living cells. In addition, laser manipulation is suitable for remote control by a computer, i.e., the system can be fully automated, unlike other methods such as micropipetting. ${ }^{12}$ As the mechanical force that can be applied by laser trapping is less than that possible in micropipetting even with the use of a vehicle such as zeolite, it is of importance to improve the efficiency. One promising approach to improve the efficiency is to spin the objects using optical force. For example, the rotary motion of a trapped object induced by optical pressure $^{13}$ could promote the efficacy of optical delivery through the membrane into the cytoplasm similar to drilling into a hard wall. Another benefit of optical trapping is that it does not require physical contact with the sample and surrounding substrates. The laser beam passes through the glass Downloaded 06 Mar 2008 to 130.54.110.22. Redistribution subject zeolite particle. or plastic transport plate glass. This feature could be beneficial under sealed conditions such as in a microflow channel or in microfabrication. Using a laser, an object can be transported to any location on the stage, whereas the application of micropipetting is much more limited. Optical tweezers can be used to transport desired particles within a fully sealed microchannel. ${ }^{14}$

In conclusion, the present results suggest that laser trapping may be useful in the field of cell biology, when combined with the use of a suitable carrier such as zeolite. It may be useful to identify or develop a vehicle that would dissolve in the cytoplasm after delivery. Future studies to identify effective carriers or vehicles for various kinds of target substrates are eagerly awaited.
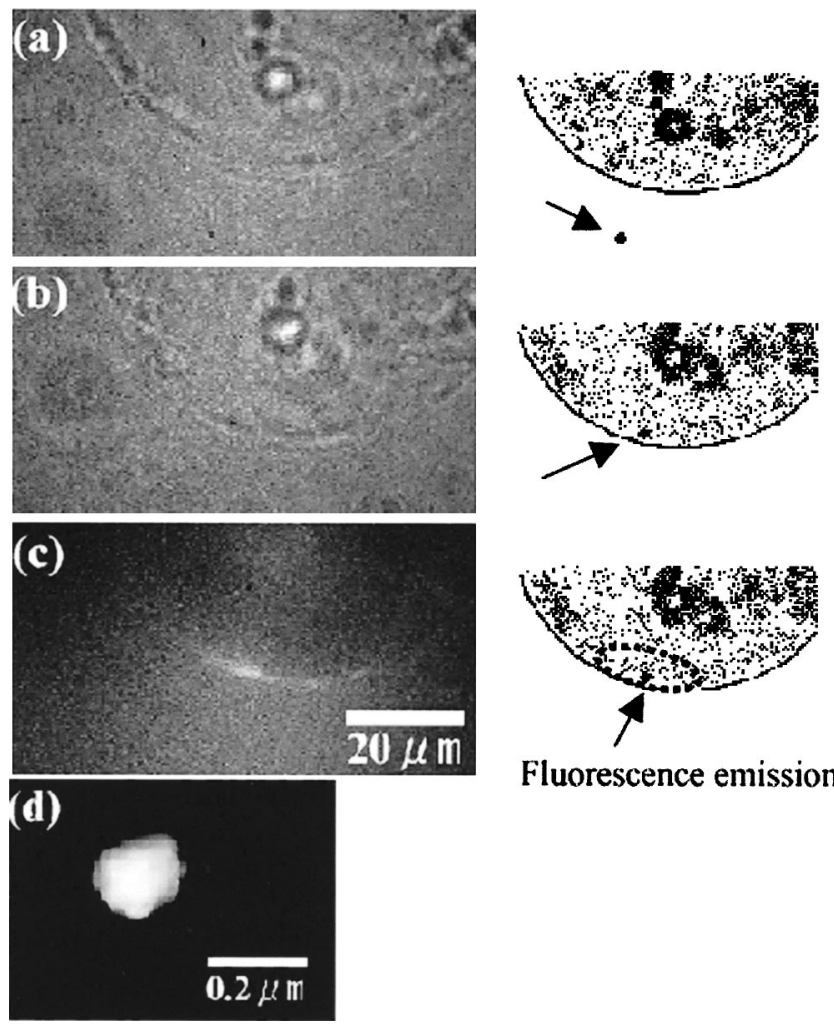

Fluorescence emission

FIG. 2. Optical delivery of a zeolite particle (marked with an arrow) doped with fura-2 into a cabbage protoplast. (a) and (b) are brightness inversion images observed by bright field microscopy, showing the process of optical delivery into the cytoplasm. (c) is the fluorescent microscopic image $1 \mathrm{~min}$ after delivery of the particle, where emission from intrinsic calcium ions complexed with fura-2 is observed. (d) Typical electron micrograph of a

AlP license or copyright; see http://apl.aip.org/apl/copyright.jsp 
The authors thank Dr. I. Moriguchi (Department of Applied Chemistry, Faculty of Engineering, Nagasaki University) for kindly providing the zeolite.

${ }^{1}$ R. Pepperkok, R. Saffrich, and W. Ansorge, Cell Biology: A Laboratory Handbook, 2nd ed. (Academic, New York, 1998), Vol. 4, p. 23.

${ }^{2}$ L. D. Spector, D. R. Goldman, and A. L. Leinwand, Cells: A Laboratory Manual (Cold Spring Harbor Laboratory, New York, 1998), Vols. 1-4.

${ }^{3}$ A. Gnirke, C. Huxley, K. Peterson, and M. V. Olson, Genomics 15, 659 (1993).

${ }^{4}$ A. Ashkin, M. J. Dziedzic, E. J. Bjorkholm, and S. Chu, Opt. Lett. 11, 288 (1986).

${ }^{5}$ K. Svoboda and S. M. Block, Annu. Rev. Biophys. Biomol. Struct. 23, 247 (1994).
${ }^{6}$ S. Chu, Science (Washington, DC, U.S.) 253, 861 (1991).

${ }^{7}$ R. Bilang, A. Kloti, M. Schrott, and I. Potrykus, Plant Molecular Biology Manual, 2nd ed. (Kluwer Academic, Dordrecht, 1994), Sec. A1.

${ }^{8}$ Y. Koyama, M. Umehara, A. Mizuno, M. Itaba, T. Yasukouchi, K. Natsume, A. Suginaka, and K. Watanabe, Bioconjugate Chem. 7, 298 (1996).

${ }^{9}$ S. M. Mel'nikov, V. G. Sergeyev, and K. Yoshikawa, J. Am. Chem. Soc. 117, 2401 (1995)

${ }^{10}$ M. Ichikawa and K. Yoshikawa, Appl. Phys. Lett. 79, 4598 (2001).

${ }^{11}$ Y. Matsuzawa, Y. Koyama, K. Hirano, T. Kanbe, A. Mizuno, and K. Yoshikawa, J. Am. Chem. Soc. 122, 2200 (2000).

${ }^{12}$ K. Schütze and G. Lahr, Nat. Biotechnol. 16, 737 (1998).

${ }^{13}$ T. Harada and K. Yoshikawa, Appl. Phys. Lett. 81, 4850 (2002).

${ }^{14}$ S. M. Nomura, Y. Yoshikawa, K. Yoshikawa, O. Dannenmuller, S. Chasserot-Golaz, G. Ourisson, and Y. Nakatani, ChemBioChem 6, 457 (2001). 